\title{
Erratum to: Inequalities of health indicators for policy makers: six hints
}

\author{
N. Speybroeck $\cdot$ S. Harper $\cdot$ D. de Savigny $\cdot$ \\ C. Victora
}

Published online: 15 August 2012

(C) Swiss School of Public Health 2012

\section{Erratum to: Int J Public Health (2012)}

\section{DOI 10.1007/s00038-012-0386-5}

In Fig. 1, right part, the data for Namibia and India was interchanged. Correctly, the Relative Inequalities are higher in Namibia than in India as described in the text.

The correct Fig. 1 reads as follows:

The online version of the original article can be found under doi:10.1007/s00038-012-0386-5.

N. Speybroeck $(\bowtie)$

Institute of Health and Society (IRSS),

Université catholique de Louvain, Brussels, Belgium

e-mail: Niko.Speybroeck@uclouvain.be

\section{S. Harper}

Department of Epidemiology,

Biostatistics and Occupational Health,

McGill University, Montreal, Canada

e-mail: sam.harper@mcgill.ca

D. de Savigny

Swiss Tropical and Public Health Institute,

University of Basel, Basel, Switzerland

e-mail: D.deSavigny@unibas.ch

C. Victora

Federal University of Pelotas, Pelotas, Brazil

e-mail: cvictora@gmail.com 
Absolute Inequalities

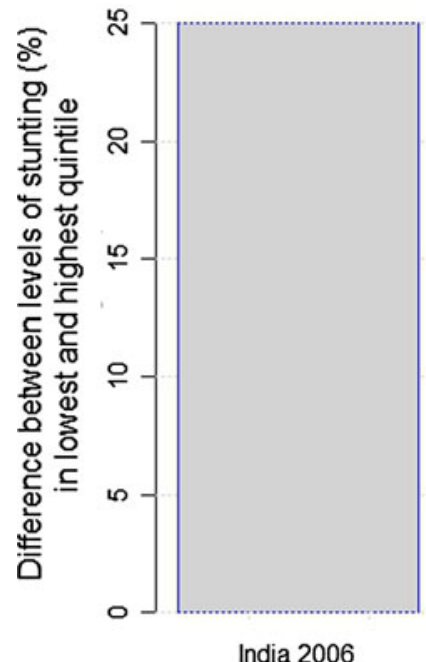

India 2006

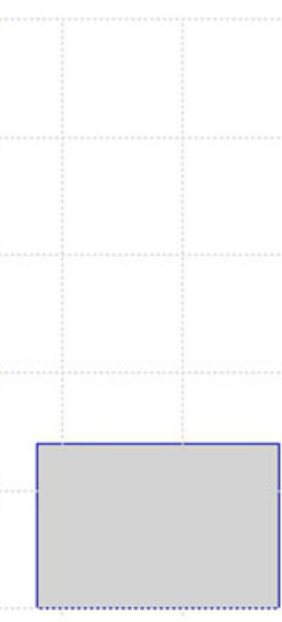

Namibia 2006

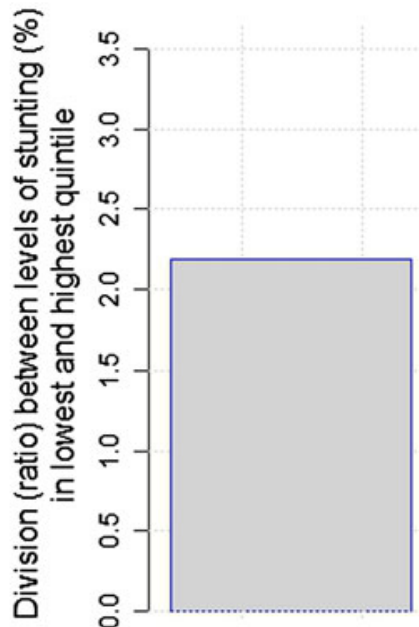

India 2006

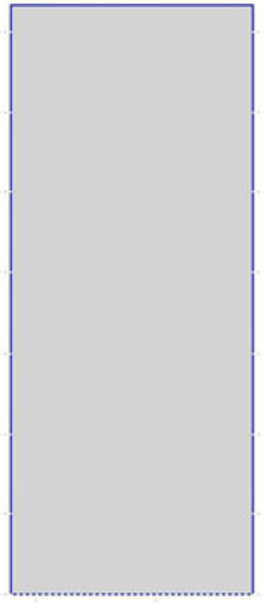

Namibia 2006

Fig. 1 Absolute (difference between levels in lowest and highest quintile) and relative inequalities (division between levels in lowest and highest income quintile) in malnutrition (stunting) in India (2006) and Namibia (2006). Source: Demographic and Health Surveys 